Editorial

\title{
Tratamiento adyuvante del cáncer de recto: cuando los árboles no dejan ver el bosque
}

Hasta mediados de los años 80 , la cirugía constituía el único abordaje terapéutico del cáncer de recto. Durante dicha década, varios estudios norteamericanos pusieron por primera vez de manifiesto la utilidad de la quimioterapia y radioterapia (RT) en el abordaje del tratamiento complementario del cáncer de recto localizado. Los datos del Gastrointestinal Study Group, NCCTG y NSABP demostraron que la adición de quimioterapia con 5-FU y RT a la cirugía suponía un incremento de control local de la enfermedad y lo mas importante, de supervivencia global ${ }^{1-3}$, estableciéndose en los EE.UU como tratamiento estándar del cáncer de recto estadio II y III. Desde entonces, el principal foco de atención de los estudios desarrollados ha sido el control local de la enfermedad. Mientras en los EE.UU, los estudios se enfocaron hacia la mejor forma de combinación del 5-FU con la RT postoperatoria (5FU bolus vs infusión continua, 5-FU modulado con leucovorin ó levamisol, etc) en Europa se desarrolló más el concepto de la RT preoperatoria. El Swedish Rectal Cancer Trial, cuyos resultados se han actualizado recientemente ${ }^{4}$, demostró que la radioterapia hipofraccionada preoperatoria (25 Gy in 5 fracciones aplicada en 1 semana) seguida de inmediata cirugía conseguía reducir la tasa de recidivas locales e incrementar la supervivencia en pacientes con cáncer rectal resecable. En este estudio, la tasa de recidivas a distancia fue similar ( $23 \%$ vs $24 \%$ ), siendo llamativa la tasa de recidivas locales en el subgrupo de pacientes tratados con cirugía sola (27\%). ¿Quiere esto significar que la utilidad de la RT preoperatoria se debe exclusivamente al efecto corrector de una cirugía inapropiada?

La respuesta a esta cuestión ha venido de la mano del desarrollo de la técnica de la excisión total del mesorrecto (TME). En manos de cirujanos expertos, con dicha técnica se han publicado tasas de recidivas locales inferiores al 10\%, que contrastan positivamente con el 15-45\% de recidivas locales publicadas en estudios previos sin dicha técnica. El Dutch Colorectal Cancer Group llevó a cabo un estudio aleatorio con 1861 pacientes con tumores rectales resecables comparando TME frente a RT seguida de TME. La tasa de recidiva local a los 2 años fue del 2.4\% para el grupo de RT, significativamente mejor que el 8.2\% obtenido con TME sola. Sin embargo, en el primer análisis de supervivencia realizado en este estudio, aunque con un seguimiento corto, no se demuestra un beneficio en supervivencia ${ }^{5}$. En este estudio, los pacientes con tumores en los 10 últimos centímetros del recto, fueron los realmente beneficiados de la introducción de la RT. La RT preoperatoria tiene su utilidad en el control local de la enfermedad independientemente de la calidad de la cirugía, pero tiene un escaso potencial para cambiar la supervivencia de los pacientes. Dos importantes interrogantes se han suscitado en los años posteriores que han dado lugar a los más recientes estudios publicados en este campo. ¿Es mejor la quimiorradioterapia que la radioterapia sola preoperatoria? ¿ Es mejor la quimiorradioterapia pre ó postoperatoria?. En cuanto a la primera cuestión, 2 estudios han arrojado similares resultados. El EORTC 22921 y el FFCD 9203 han demostrado que la adición de bolus 5-FU/LV a la RT preoperatoria supone un incremento en las respuestas completas patológicas, y en el control local de la enfermedad pero no observan ventajas en la supervivencia ${ }^{6,7}$. La segunda cuestión ha sido el objetivo del estudio CAO/ARO/AIO-94 del German Rectal Cancer Study Group. La quimiorradioterapia preoperatorio reduce significativamente las recidivas locales (6\% vs $13 \%$ ) con respecto a la postoperatoria pero nuevamente no cambia la supervivencia global de los pacientes $(76 \% \text { vs } 74 \%)^{8}$. 


\section{J. Sastre}

Con todos estos datos encima de la mesa, podemos afirmar que una correcta cirugía mediante TME es importante, la radioterapia preoperatoria es preferible a la postoperatoria, y la adición de quimioterapia a la RT preoperatoria es útil, pero todo ello redunda en un buen control local de la enfermedad. Sin duda, suponen importantes avances de la oncología, ya que evitar la morbilidad de la recidiva local del cáncer de recto debe ser por sí mismo un objetivo. Sin embargo, tras 20 años de ensayos clínicos no se ha conseguido incrementar la supervivencia de los pacientes con cáncer rectal resecable. ¿Dónde pueden estar las claves en el fracaso en mejorar la supervivencia?. Sin duda alguna, en el control de la enfermedad sistémica micrometastásica. Si analizamos este aspecto en los diferentes ensayos evaluados y los comparamos con la evolución experimentada en este campo en el cáncer de colon podemos acercarnos a los puntos clave del citado fracaso. En cáncer de colon, durante el mismo periodo de tiempo, los estudios de tratamiento quimioterápico adyuvante han demostrado que la duración óptima del mismo debe ser de 6 meses. Un reciente análisis del SEER Medicare database, en pacientes con cáncer de colon estadio III tratados con 5-FU adyuvante, sugiere que los pacientes tratados durante un periodo de tiempo inferior a 5 meses tienen una significativa menor supervivencia que aquellos que reciben de 5-7 meses de tratamiento adyuvante ${ }^{9}$. En los estudios de cáncer de recto es una constante el escaso periodo de tiempo de quimioterapia adyuvante, limitándose en la mayoría a 4 meses de tratamiento con 5-FU/LV. Aunque la dosis de 5-FU, en los esquemas mensuales asociados a bajas dosis de LV, han variado en los estudios de adyuvancia en colon, en ningún estudio se utilizan dosis tan bajas de $350 \mathrm{mg} / \mathrm{m} 2 /$ día x 5 días utilizadas en el estudio de la EORTC 22921, en el cual 4 ciclos a estas dosis son insuficientes para el control sistémico de la enfermedad. Los estudios en cáncer de recto han incluido estadios II y III, no estando a día de hoy totalmente claro el beneficio de 5-FU/LV en la supervivencia en los estadios II, lo que puede contribuir a minimizar el potencial efecto beneficioso de la quimioterapia adyuvante. Por último, en cáncer de colon se ha demostrado el beneficio de nuevos fármacos activos en el tratamiento adyuvante, como el oxaliplatino, especialmente en los subgrupos más desfavorables (estadio III-N2). En el estudio CAO/ARO/AIO, los pacientes en tratamiento preoperatorio que tras la cirugía mantenían afectación ganglionar presentaban un pronóstico muy desfavorable, con tan solo un $46 \%$ de supervivencia libre de enfermedad a 5 años. Probablemente, esquemas de quimioterapia basados exclusivamente en la modulación de 5-FU son insuficientes para el control sistémico de dicha subpoblación.

En el futuro, es importante diseñar estrategias de tratamiento globales destinadas a incrementar el control sistémico y local de la enfermedad, con modernos fármacos activos en la enfermedad metastásica como los nuevos anticuerpos monoclonales frente al EGFR y VEGF, y con una duración óptima del tratamiento sistémico global, bien sea administrado preoperatoriamente, postoperatoriamente o una combinación de ambas modalidades. Los árboles no nos deben impedir ver el bosque.

Dr. Javier Sastre

Servicio de Oncología Médica Hospital Clínico Univ. San Carlos Madrid (España) 


\section{Bibliografía}

1. Gastrointestinal Tumor Study Group. Prolongation of the disease-free interval in surgically treated rectal carcinoma. N Engl J Med 1985, 312:1465-1472.

2. Douglass HO Jr, Moertel CG, Mayer RJ et al. Survival after postoperative combination treatment of rectal cancer. N Engl J Med 1986, 315:1294-1295

3. Wolkmark N, Wieand HS, Hyams DM et al. Randomized trial of postoperative adjuvant chemotherapy with or without radiotherapy for carcinoma of the rectum: National Surgical Adjuvant Breast and Bowel Project Protocolo R-02. J Nat Cancer Inst 2000,92:388-396.

4. Swedish Rectal Cancer Trial. Improved survival with preoperative radiotherapy in respectable rectal cancer. N Engl J Med 1997, 336:980-987.

5. Kapiteijn E, Marijnen CAM, Nagtegaal ID et al. Preoperative radiotherapy combined with total mesorectal excision for resectable rectal cancer. N Engl J Med 2001, 345:638-646.

6. Bosset J, Calais G, Mineur L et al. Preoperative radiation (Preop RT) in rectal cancer: effect and timing of additional chemotherapy (CT) 5-year results of the EORTC 22921 trial. J Clin Oncol 2005, 23:247s

7. Gerard J, Romestaing P, Bonnetain F et al. Preoperative chemoradiotherapy (CT-RT) improves local control in T3-4 rectal cancers: Results of the FFCD 9203 randomised trial. Int J Radiat Oncol Biol Phys 2005, 63:S2.

8. Sauer R, Becker H, Hohenberger W et al. Preoperative versus postoperative chemoradiotherapy for rectal cancer. N Engl J Med 2004,351:1731-1740.

9. Neugut AI, Matasar M, Wang X et al. Duration of adjuvant chemotherapy for colon cancer and survival among the elderly. J Clin Oncol 2006, 24:2368-2375. 